\title{
Widely tunable twin-guide laser diodes with large continuous tuning range
}

\author{
R. Todt ${ }^{1}$, T. Jacke ${ }^{1}$, R. Meyer ${ }^{1}$, R. Laroy ${ }^{2}$, G. Morthier ${ }^{2}$ and M.-C. Amann ${ }^{1}$ \\ ${ }^{1}$ Walter Schottky Institut, Technische Universität München, 85748 Garching, Germany \\ e-mail: todt@wsi.tum.de \\ ${ }^{2}$ Ghent University, Department of Information Technology, Sint-Pietersnieuwstraat 41, 9000 Gent, Belgium
}

\begin{abstract}
Widely tunable twin-guide laser diodes providing an SMSR and an output power above $35 \mathrm{~dB}$ and $10 \mathrm{~mW}$, respectively, over a $40 \mathrm{~nm}$ tuning range are presented. Continuous tuning ranges as large as $8.2 \mathrm{~nm}$ are achieved.
\end{abstract}

\section{Introduction}

With the rapid growth of the global internet traffic, widely tunable laser diodes with tuning ranges of several tens of nanometers are becoming increasingly important to realize agile optical networks. Monolithic tunable devices are especially well suited for communication applications due to their fast tuning speed and their excellent reliability. In the past, several DBR-type widely tunable lasers based on the Vernier effect have been demonstrated [1-4]. However, requiring at least three tuning currents to set the emission wavelength, the characterization and control of these devices is complicated, time-consuming and, therefore, expensive. Recently, a novel Vernier-effect-based tunable laser, the so-called sampled grating tunable twin-guide (SG-TTG) laser, offering a simplified wavelength control was suggested [5] and experimentally demonstrated [6]. This paper reports the recent progress on the SG-TTG laser.

\section{Device structure}

The SG-TTG laser is based on the original DFB-TTG laser, which has achieved record continuous tuning ranges of up to $9.3 \mathrm{~nm}$ by carrier injection [7]. In the SG-TTG laser (Fig. 1), the uniform DFB grating of the DFB-TTG laser is replaced by two SG's that can be tuned independently from each other via the tuning currents $I_{\mathrm{t}, \mathrm{F}}$ and $I_{\mathrm{t}, \mathrm{R}}$ and, thus, enable Vernier-effect-tuning. Being essentially a transversely integrated DFB-like laser, a phase tuning section as required for the longitudinally integrated DBR-type tunable lasers is not required in the SG-TTG laser. Due to the large continuous tuning range and the need for only two tuning currents, the SG-TTG laser offers a comparatively simple wavelength control. Moreover, initial measurements indicate that the SG-TTG laser has promising prospects for direct modulation at $10 \mathrm{GHz}[8]$.

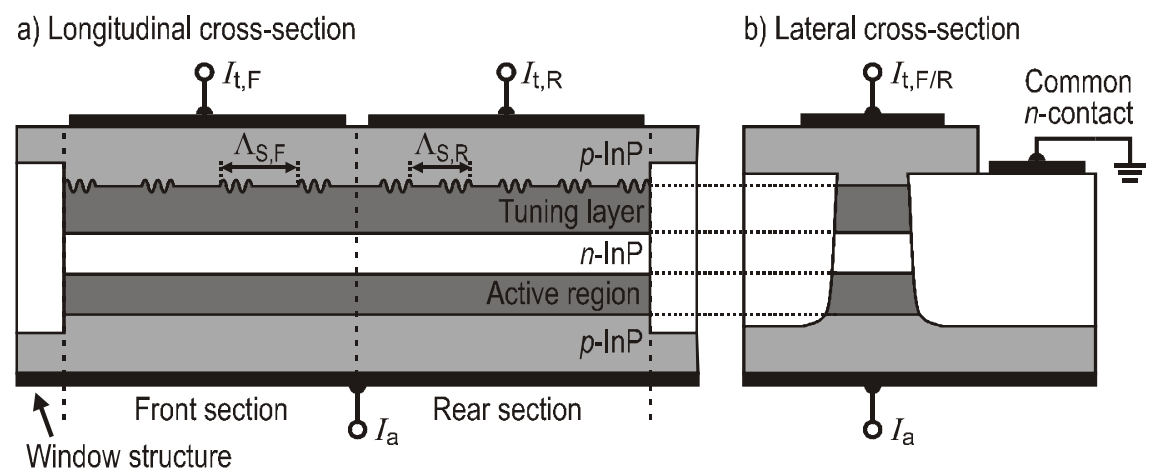

Fig. 1: Schematic drawings of the sampled grating tunable twin-guide (SG-TTG) laser diode. The devices are fabricated in the GaInAsP/InP material system employing standard buried heterostructure technology. Window structures in combination with antireflection coatings are used to suppress spurious facet reflections that would otherwise interfere with the SG reflections [9].

\section{Results}

Fig. 2a shows the wavelength map of an SG-TTG laser obtained by sweeping both tuning currents in a non-linear way up to a maximum current of $45 \mathrm{~mA}$. Within each of the various supermodes, the emission wavelength of the laser changes continuously by up to $8.2 \mathrm{~nm}$. Fig. $2 \mathrm{~b}$ illustrates the wavelength range covered by the nine main 
supermodes. Over a wavelength range of $41 \mathrm{~nm}$ (from 1520.5 to $1561.5 \mathrm{~nm}$ ), the SMSR and output power remains above $35 \mathrm{~dB}$ and $10 \mathrm{~mW}$, respectively, and the output power variation amounts to $3.8 \mathrm{~dB}$.
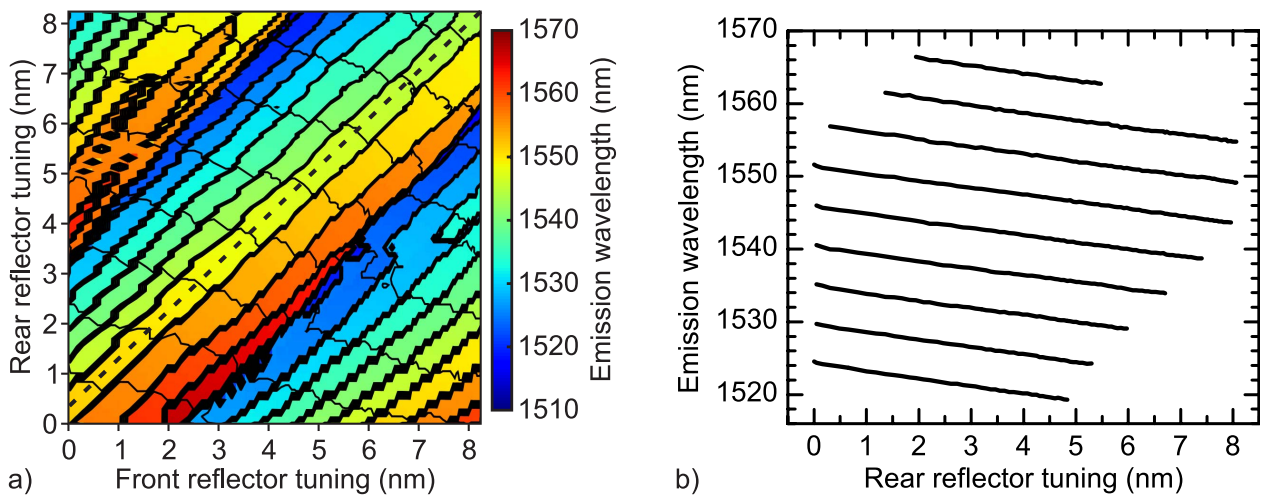

Fig. 2: a) Wavelength map of an SG-TTG laser diode. The various supermodes are enclosed by bold lines. Thin lines within the supermodes indicate iso-wavelength contours that are spaced in $1.0 \mathrm{~nm}$-intervals and should not be confused with longitudinal mode jumps that would occur in DBR-type tunable lasers. Within each supermode, the wavelength changes continuously (as illustrated in Fig. 3 for the central supermode) and the iso-wavelength contours are shown as a visual aid for determining the corresponding continuous tuning range. b) Emission wavelength versus rear reflector tuning for the main supermodes, illustrating full coverage of a tuning range of more than $40 \mathrm{~nm}$.

Details of the tuning behavior within a typical supermode are revealed in Fig. 3, which illustrates the continuous wavelength tuning obtained by changing both tuning currents simultaneously. While tuning along a supermode, the SMSR and the output power decrease almost linearly. The achieved continuous tuning range of up to $8.2 \mathrm{~nm}$ significantly exceeds that of DBR-type widely tunable laser diodes and, thereby, facilitates an efficient and easy device characterization and control.
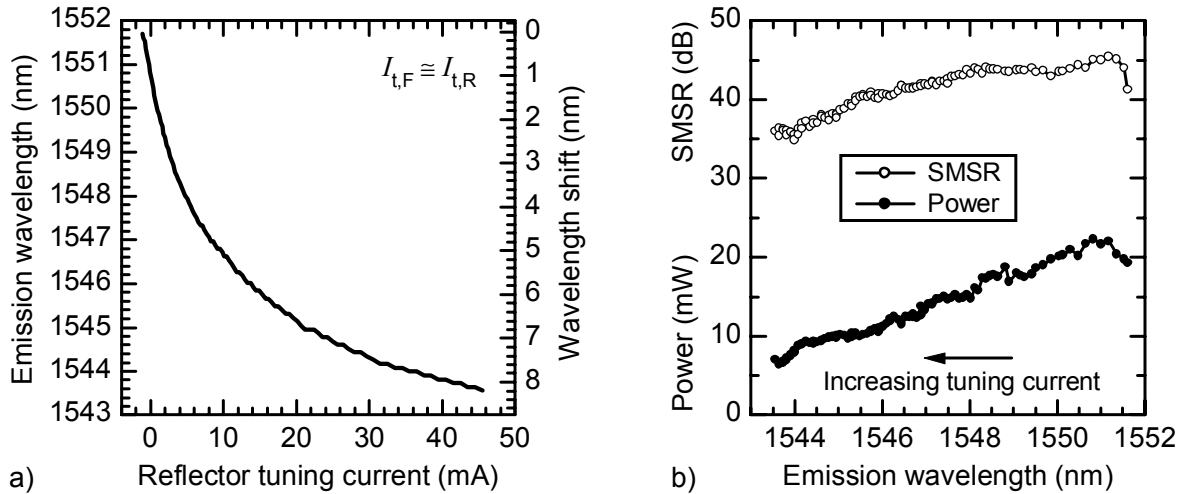

Fig. 3: Tuning characteristics of the central supermode (indicated as dashed line in Fig. 2a). a) Emission wavelength and wavelength shift versus tuning current. b) Variation of output power and SMSR as function of the emission wavelength.

\section{Conclusion}

In conclusion, state-of-the-art performance of SG-TTG laser diodes offering optical characteristics comparable to longitudinally integrated DBR lasers has been demonstrated. The large continuous tuning range of this device as well as its simple wavelength control requiring only two tuning currents are unmatched hitherto.

[1] V. Jayaraman et al., IEEE J. Quantum Electron. 29, 1824 - 1834, June 1993.

[2] Y. Tohmori et al., IEEE J. Quantum Electron. 29, 1817 - 1823, June 1993.

[3] D. C. J. Reid et al., Proc. Optical Fiber Communication Conference (OFC), pp. 541 - 543, paper ThV5, 2002.

[4] J.-O.Wesström et al., Proc. Optical Fiber Communication Conference (OFC), pp. 389 - 391, paper TuE2, 2004.

[5] G. Morthier et al., IEEE Photon. Technol. Lett. 13, pp. 1052 - 1054, Oct. 2001.

[6] R. Todt et al., IEEE Photon. Technol. Lett. 17, pp. 2514 - 2516, Sep. 2005.

[7] R. Todt et al., Electron. Lett. 41, pp. 1063 - 1065, Dec. 2005.

[8] R. Laroy et al., IEEE Photon. Technol. Lett. 18, 2006 (accepted for publication).

[9] R. Todt et al., IEEE Photon. Technol. Lett. 17, pp. 2520 - 2522, Sep. 2005. 
